\title{
ABOUT SOME SYMMETRIES OF NEGATION
}

\author{
BRIGITTE HÖSLI AND GERHARD JÄGER
}

\begin{abstract}
This paper deals with some structural properties of the sequent calculus and describes strong symmetries between cut-free derivations and derivations, which do not make use of identity axioms. Both of them are discussed from a semantic and syntactic point of view.

Identity axioms and cuts are closely related to the treatment of negation in the sequent calculus. so the results of this article explain some nice symmetries of negation.
\end{abstract}

§1. Introduction. Gentzen's sequent calculus LK is an extremely powerful tool in the syntactic tradition of logic. In a way, the sense of each propositional connective and quantifier is provided by the corresponding logical rules which regulate its introduction on the left- and right-hand side of a sequent. These introduction rules respect perfect left/right symmetries, and these symmetries are the actual reason that it is possible to prove strong results like cut elimination.

To give an example, one may consider the two rules for introducing the negation symbol:

$$
\frac{\Gamma \supset \Delta, A}{\Gamma, \neg A \supset \Delta} \quad(\mathrm{l} \neg) \quad \frac{\Gamma, A \supset \Delta}{\Gamma \supset \Delta, \neg A} \quad(\mathrm{r} \neg) .
$$

Similar rules exist for all other propositional connectives and the quantifiers. The only purpose - but a crucial one - of these introduction rules is the building up of complex formulas from more elementary subformulas according to the principles inherent in the usual truth table interpretation. ${ }^{1}$ Hence, they reflect the denotational or algebraic aspects of the respective logical symbols and serve as perfect bookkeeping tools.

However, there exists a significant difference between the treatment of negation and the other connectives like, e.g. conjunction. The rules for negation are not confined to the introduction rules $(l \neg)$ and $(r \neg)$ but comprise in addition the following identity axioms and cut rules

$$
\Gamma, A \supset \Delta, A \quad \text { (id) } \quad \frac{\Gamma \supset \Delta, A \Gamma, A \supset \Delta}{\Gamma \supset \Delta} \quad \text { (cut) }
$$

which add some features of communication between occurrences of a formula $A$ in the premise and consequence of a sequent: The identity axioms express that $A$ on the left is at least as strong as $A$ on the right; cuts, on the other hand, state the converse, i.e. that $A$ on the right is at least as strong as $A$ on the left. Hence,

\footnotetext{
Received May 7, 1993.

${ }^{1}$ Existential and universal quantifiers can be interpreted as uniform infinitary disjunctions and conjunctions, respectively. 
the actual meaning of negation is implicit in (the interplay between) the identity axioms and cut rules. One can say that in a certain sense the negation introduction rules $(l \neg)$ and $(\mathbf{r} \neg)$ deal with the statical aspects of negation, whereas the identity axioms (id) and the cut rules (cut) cope with the dynamical aspects of negation.

Gentzen proved that the cut rules are redundant in the pure sequent calculus, i.e. in the sequent calculus without nonlogical axioms. Cut elimination fails, on the other hand, if sufficiently complicated extra axioms are permitted.

Girard [1] contains a detailed analysis of the cut-free sequent calculus with extra axioms. His approach is based on three-valued semantics and makes use of some interesting versions of three-valued logic. One of the central results states that the closed sequent $\Gamma \supset \Delta$ is cut-free provable from the theory $T$ if and only if $\Gamma \supset \Delta$ is a semantical consequence of $T$ with respect to so called Schütte valuations; the precise formulation of this theorem is given below (cf. Theorem 4).

Sequent calculi without identity axioms but additional nonlogical axioms and nonlogical rules have gained some interest only recently. They play an important role in the proof-theoretic analysis of logic programming and in connection with the so called negation as failure rule (cf. e.g. Jäger [3] and Stärk [7]). It has been shown that they are closely related to the three-valued completions of logic programs in the sense of Kunen [5], and that they form a sound and complete basis for a deductive approach to SLDNF-resolution.

In this paper we do not restrict ourselves to logic programming but take a broader and more elementary point of view. Conceptually, the focus is put on some very strong symmetries between the identity axioms and the cut rules. The main technical achievements are results for the sequent calculus without identity axioms which are dual to Girard's result for the cut-free sequent calculus, always with nonlogical extra axioms.

After introducing the basic terminology we consider some semantical aspects of calculi without cut rule or identity axioms. We introduce weak and strong Schütte valuations and prove soundness and completeness for these calculi with respect to the appropriate Schütte valuations. Then we turn to syntactical considerations and consider cut-free and identity-free derivations from this point of view.

$\S 2$. Restrictions of the sequent calculus. Let $\mathscr{L}$ be an arbitrary first-order language with free variables $u, v, w, u_{1}, v_{1}, w_{1}, \ldots$, bound variables $x, y, z, x_{1}, y_{1}$, $z_{1}, \ldots$, function and relation symbols, and $\neg, \vee, \wedge, \exists$, and $\forall$ as logical connectives and quantifiers. The terms $a, b, c, a_{1}, b_{1}, c_{1}, \ldots$ and formulas $A, B, C, A_{1}, B_{1}$, $C_{1}, \ldots$ of $\mathscr{L}$ are defined as usual.

The capital Greek letters $\Gamma, \Delta, \Pi, \Sigma, \Gamma_{1}, \Delta_{1}, \Pi_{1}, \Sigma_{1} \ldots$ denote finite sequences of $\mathscr{L}$ formulas, and $\mathscr{L}$ sequents are expressions of the form $\Gamma \supset \Delta$. A (possible infinite) set of $\mathscr{L}$ sequents which is closed under substitution ${ }^{2}$ is often called an $\mathscr{L}$ theory.

Gentzen's sequent calculus LK is described in detail for example in Girard [1] and Takeuti [8]. For completeness we now repeat the axioms and rules of LK.

\footnotetext{
${ }^{2}$ This means that $\Gamma(a) \supset \Delta(a) \in T$ if $\Gamma(u) \supset \Delta(u) \in T$.
} 
I. Structural Rules.

$$
\begin{aligned}
& \text { Weakening } \\
& \frac{\Gamma \supset \Delta}{\Gamma, A \supset \Delta} \quad(\mathrm{lW}) \quad \frac{\Gamma \supset \Delta}{\Gamma \supset \Delta, A} \quad(\mathrm{rW}) \\
& \text { Exchange } \\
& \frac{\Gamma_{1}, A, B, \Gamma_{2} \supset \Delta}{\Gamma_{1}, B, A, \Gamma_{2} \supset \Delta} \quad \text { (IE) } \quad \frac{\Gamma \supset \Delta_{1}, A, B, \Delta_{2}}{\Gamma \supset \Delta_{1}, B, A, \Delta_{2}} \\
& \text { Contraction } \\
& \frac{\Gamma, A, A \supset \Delta}{\Gamma, A \supset \Delta} \quad \text { (1C) } \quad \frac{\Gamma \supset \Delta, A, A}{\Gamma \supset \Delta, A} \quad \text { (rC) }
\end{aligned}
$$

II. LOGICAL RULES.

Negation

$$
\begin{aligned}
& \frac{\Gamma \supset \Delta, A}{\Gamma, \neg A \supset \Delta} \quad(1 \neg) \quad \frac{\Gamma, A \supset \Delta}{\Gamma \supset \Delta, \neg A} \quad(\mathrm{r} \neg) \\
& \left.\left.\frac{\Gamma, A(u) \supset \Delta}{\Gamma, \exists x A(x) \supset \Delta} \quad \text { (1 }\right) \quad \frac{\Gamma \supset \Delta, A(a)}{\Gamma \supset \Delta, \exists x A(x)} \quad \text { (r } \exists\right) .
\end{aligned}
$$

Universal quantifier

$$
\frac{\Gamma, A(a) \supset \Delta}{\Gamma, \forall x A(x) \supset \Delta} \quad(1 \forall) \quad \frac{\Gamma \supset \Delta, A(u)}{\Gamma \supset \Delta, \forall x A(x)} \quad(\mathrm{r} \forall)
$$

In the rules, $(1 \exists)$ and $(\mathrm{r} \forall)$ are subject to the variable condition that the free variable $u$ must not occur in the conclusion.

III. IDENTITY AXIOMS AND CUT RULES.

$$
\Gamma, A \supset \Delta, A \quad \text { (id) } \quad \frac{\Gamma \supset \Delta, A \quad \Gamma, A \supset \Delta}{\Gamma \supset \Delta} \quad \text { (cut) }
$$

Let $T$ be an $\mathscr{L}$ theory. Then the notion $T \vdash \Gamma \supset \Delta$ is used to express that the sequent $\Gamma \supset \Delta$ is provable from $T$; it is inductively defined as follows:

(1) If $\Gamma \supset \Delta$ is an identity axiom or an element of $T$, then we have $T \vdash \Gamma \supset \Delta$.

(2) If $T \vdash \Gamma_{i} \supset \Delta_{i}$ for every premise $\Gamma_{i} \supset \Delta_{i}$ of a structural, a logical rule or a cut, then we have $T \vdash \Gamma \supset \Delta$ for the conclusion $\Gamma \supset \Delta$ of this rule.

We write $T \vdash_{0} \Gamma \supset \Delta$ if the sequent $\Gamma \supset \Delta$ is cut-free provable form $T$, i.e., if there is a proof using only the identity axioms, the elements of $T$, the structural 
rules, and the logical rules. $T \Vdash \Gamma \supset \Delta$, on the other hand, means that the sequent $\Gamma \supset \Delta$ is provable form $T$ by a proof which does not make use of the identity axioms. In this case we say that $\Gamma \supset \Delta$ is identity-free provable from $T$. Finally, the notation $T \Vdash_{0} \Gamma \supset \Delta$ expresses that $\Gamma \supset \Delta$ is cut-free and identity-free provable from $T$.

The omission of the identity axioms may appear strange at first sight. And indeed, if we do not have extra nonlogical axioms, then there are no identityfree provable sequents. In general, however, the collection of sequents which are identity-free provable from a (nonempty) theory $T$ is quite interesting. Some general considerations concerning identity-free sequent calculi are presented in a separate article; specific applications of identity-free sequent calculi in logic programming are described in Jäger [3] and Stärk [7].

In the following we study some semantical and syntactical properties of cut-free and identity-free provability. The main emphasis is put on pointing out the strong symmetries and dualities between both restrictions of the sequent calculus. These considerations provide some further evidence for the fact that the identity axioms and the cuts reflect the two faces of the meaning of negation.

§3. Semantical considerations. We begin this section by recapitulating the soundness and completeness result for the cut-free sequent calculus. A detailed presentation of the main work on the semantics of the cut-free sequent calculus and related topics is given in Girard [1]. An important step in his approach is to consider threevalued rather than two-valued valuations: A third truth value $\mathbf{u}$ is added to $\mathbf{t}$ (true) and $\mathbf{f}$ (false) which can be vaguely described as undefined or undetermined.

Definition 1. A three-valued valuation for $\mathscr{L}$ is a function $V$ which assigns a truth value $V(A) \in\{\mathbf{t}, \mathbf{f}, \mathbf{u}\}$ to all $\mathscr{L}$ formulas $A$.

Soundness and completeness of the cut-free sequent calculus is formulated in [1] with respect to so called Schütte valuations. In this paper we will also need a dual concept in order to show soundness and completeness of the identity-free sequent calculus so that we use the more specific notion of weak Schütte valuations instead.

Definition 2. A three-valued valuation $V$ for $\mathscr{L}$ is called a weak Schütte valuation if the following conditions are satisfied:

(W.1) If $V(\neg A)=\mathbf{f}$, then $V(A)=\mathbf{t}$;

if $V(\neg A)=\mathbf{t}$, then $V(A)=\mathbf{f}$.

(W.2) If $V(A \vee B)=\mathbf{f}$, then $V(A)=\mathbf{f}$, and $V(B)=\mathbf{f}$;

if $V(A \vee B)=\mathbf{t}$, then $V(A)=\mathbf{t}$ or $V(B)=\mathbf{t}$.

(W.3) If $V(A \wedge B)=\mathbf{f}$, then $V(A)=\mathbf{f}$ or $V(B)=\mathbf{f}$;

if $V(A \wedge B)=\mathbf{t}$, then $V(A)=\mathbf{t}$ and $V(B)=\mathbf{t}$.

(W.4) If $V(\exists x A(x))=\mathbf{f}$, then $V(A(a))=\mathbf{f}$ for all terms $a$ of $\mathscr{L}$;

if $(\exists x A(x))=\mathbf{t}$, then $V(A(u))=\mathbf{t}$ for some free variable $u$ of $\mathscr{L}$.

(W.5) If $V((\forall x A(x))=\mathbf{f}$, then $V(A(u))=\mathbf{f}$ for some free variable $u$ of $\mathscr{L}$;

if $V(\forall x A(x))=\mathbf{t}$, then $V(A(a))=\mathbf{t}$ for all terms $a$ of $\mathscr{L}$.

It is important to observe that in the case of a weak Schütte valuation the value of a complex formula is not determined by the values of its subformulas; this may 
happen even if all subformulas of $A$ take values in $\{\mathbf{t}, \mathbf{f}\}{ }^{3}$ Weak Schütte valuations only propagate the definite truth values $t$ and $f$ from compound formulas to (some of) their subformulas.

The next step is to introduce a notion of semantical consequence with respect to weak Schütte valuations. This is done by means of so called weak models, in contrast to strong models which will be used later (cf. Lemma 6).

Definition 3. Let $V$ be a three-valued valuation for $\mathscr{L}, \Gamma \supset \Delta$ an $\mathscr{L}$ sequent, and $T$ an $\mathscr{L}$ theory.

(1) $V$ is called a weak model of $\Gamma \supset \Delta$ if $V(A) \neq \mathbf{t}$ for some $A$ in $\Gamma$ or $V(B) \neq \mathbf{f}$ for some $B$ in $\Delta$; in this case we write $V \vDash_{w} \Gamma \supset \Delta$.

(2) $V$ is called a weak model of $T$ if $V \vDash_{w} \Pi \supset \Sigma$ for all $\Pi \supset \Sigma$ in $T$; in this case we write $V F_{w} T$.

(3) $\Gamma \supset \Delta$ is called a weak consequence of $T$ if every weak Schütte valuation which is a weak model of $T$ is also a weak model of $\Gamma \supset \Delta$; in this case we write $T \vDash_{w} \Gamma \supset \Delta$.

The following result is due to Girard and establishes soundness and completeness of the cut-free sequent calculus with respect to the weak Schütte valuations. A detailed proof is given in [1].

THEOREM 4 (weak soundness and completeness). We have for all $\mathscr{L}$ theories $T$ and all $\mathscr{L}$ sequents $\Gamma \supset \Delta$ :

$$
T \vdash_{0} \Gamma \supset \Delta \Leftrightarrow T \vDash_{w} \Gamma \supset \Delta .
$$

Now we turn to the identity-free sequent calculus. Instead of using weak Schütte valuations we work with strong Schütte valuations, i.e., three-valued valuations so that the definite truth values do not propagate from compound formulas to their subformulas but from (some of) the subformulas of a compound formula to the compound formula.

Definition 5. A three-valued valuation $V$ for $\mathscr{L}$ is called a strong Schütte valuation if the following conditions are satisfied:

(S.1) If $V(A)=\mathbf{f}$, then $V(\neg A)=\mathbf{t}$; if $V(A)=\mathbf{t}$, then $V(\neg A)=\mathbf{f}$.

(S.2) If $V(A)=\mathbf{f}$ and $V(B)=\mathbf{f}$, then $V(A \vee B)=\mathbf{f}$;

if $V(A)=\mathbf{t}$ or $V(B)=\mathbf{t}$, then $V(A \vee B)=\mathbf{t}$.

(S.3) If $V(A)=\mathbf{f}$ or $V(B)=\mathbf{f}$, then $V(A \wedge B)=\mathbf{f}$;

if $V(A)=\mathbf{t}$ and $V(B)=\mathbf{t}$, then $V(A \wedge B)=\mathbf{t}$.

(S.4) If $V(A(u))=\mathbf{f}$ for all free variables $u$ of $\mathscr{L}$, then $V(\exists x A(x))=\mathbf{f}$; if $V(A(a))=\mathbf{t}$ for some term $a$ of $\mathscr{L}$, then $V(\exists x A(x))=\mathbf{t}$.

(S.5) If $V(A(a))=\mathbf{f}$ for some term $a$ of $\mathscr{L}$, then $V(\forall x A(x))=\mathbf{f}$;

if $V(A(u))=\mathbf{t}$ for all free variables $u$ of $\mathscr{L}$, then $V(\forall x(A(x))=\mathbf{t}$.

Also in this case the values of the subformulas of a formula $A$ do not necessarily determine the value of $A$. However, if the values of all immediate subformulas of $A$ belong to $\{\mathbf{t}, \mathbf{f}\}$, then $V(A)$ is determined and belongs to $\{\mathbf{t}, \mathbf{f}\}$ as well.

For two-valued valuations, i.e. valuations which never take the value $\mathbf{u}$, the situation is particularly simple: A two-valued valuation is a weak Schütte valuation if and only if it is a strong Schütte valuation.

\footnotetext{
${ }^{3}$ If $V(B)=V(C)=\mathbf{t}$, then $V(B \wedge C)$ can take the values $\mathbf{t}, \mathbf{u}$.
} 
Since every three-valued valuation is a weak model of the identity axioms, weak models are bound to be useless in the context of identity-free systems. Hence, we replace them by the in this sense more restrictive strong models and base the semantic interpretation of the identity-free sequent calculus on those.

Definition 6. Let $V$ be a three-valued valuation for $\mathscr{L}, \Gamma \supset \Delta$ an $\mathscr{L}$ sequent, and $T$ an $\mathscr{L}$ theory.

(1) $V$ is called a strong model of $\Gamma \supset \Delta$ if $V(A)=\mathbf{f}$ for some $A$ in $\Gamma$ or $V(B)=\mathbf{t}$ for some $B$ in $\Delta$; in this case we write $V \vDash_{s} \Gamma \supset \Delta$.

(2) $V$ is called a strong model of $T$ if $V \vDash_{s} \Pi \supset \Sigma$ for all $\Pi \supset \Sigma$ in $T$; in this case we write $V \vDash_{s} T$.

(3) $\Gamma \supset \Delta$ is called a strong consequence of $T$ if every strong Schütte valuation which is a strong model of $T$ is also a strong model of $\Gamma \supset \Delta$; in this case we write $T \vDash_{s} \Gamma \supset \Delta$.

To point out the distinction between weak and strong models let us consider a sequent $\supset \Delta$. Then $V$ is a weak model of $\supset \Delta$ provided that $V(B) \neq \mathbf{f}$ for some $B$ in $\Delta$. If $V$ is a strong model of $\supset \Delta$, then we even know that there is a $B$ in $\Delta$ so that $B(V)=\mathbf{t}$.

If $V(A)=\mathbf{u}$ for some three-valued valuation $V$ and $\mathscr{L}$ formula $A$, then $V$ is not a strong model of $A \supset A$. Hence, the identity axioms are not generally satisfied. However, the conditions S.1-S.5 of strong Schütte valuations preserve the validity of the structural rules, the logical rules, and the cut rules with respect to strong models so that one obtains the following soundness result.

THEOREM 7 (Strong soundness). We have for all $\mathscr{L}$ theories $T$ and all $\mathscr{L}$ sequents $\Gamma \supset \Delta$ :

$$
T \Vdash \Gamma \supset \Delta \Rightarrow T \vDash_{s} \Gamma \supset \Delta .
$$

This theorem is proved by straightforward induction on the length of the derivation so that we can omit the details. In order to show the converse direction, i.e. strong completeness, we follow the pattern of Schütte's completeness proofs in [6] and make use of so called deduction chains.

For the following definitions we need some auxiliary notation. First we assume that we are given some (arbitrary) enumerations

$$
s_{0}, s_{1}, s_{2}, \ldots, \quad t_{0}, t_{1}, t_{2}, \ldots, \quad \text { and } F_{0}, F_{1}, F_{2}, \ldots
$$

of the free variables, terms and formulas of $\mathscr{L}$, respectively. Second, if $\Gamma$ is a finite sequence of $\mathscr{L}$ formulas, then we write $\operatorname{set}(\Gamma)$ for the set of its components. Third, an $\mathscr{L}$ sequent $\Gamma \supset \Delta$ belongs to the $W$-closure of an $\mathscr{L}$ theory $T$ (closure of $T$ under weakening) if there exists a sequent $\Pi \supset \Sigma$ in $T$ so that $\operatorname{set}(\Pi) \subset \operatorname{set}(\Gamma)$ and $\operatorname{set}(\Sigma) \subset \operatorname{set}(\Delta)$

Now assume that $T$ is an $\mathscr{L}$ theory and $\Gamma \supset \Delta$ an $\mathscr{L}$ sequent. Then a $\mathrm{D}$-chain (deduction chain) for $\Gamma \supset \Delta$ with respect to $T$ is a sequence

$$
\Gamma_{0} \supset \Delta_{0}, \Gamma_{1} \supset \Delta_{1}, \ldots
$$

of $\mathscr{L}$ sequents which is formed as follows:

(D.1) The initial sequent $\Gamma_{0} \supset \Delta_{0}$ of the D-chain is the sequent $\Gamma \supset \Delta$. 
(D.2) If a sequent $\Gamma_{n} \supset \Delta_{n}$ belongs to the W-closure of $T$, then it is the last sequent of the D-chain. Then we say that the D-chain has length $n$.

(D.3) If $n=3 m$ and $\Gamma_{n} \supset \Delta_{n}$ does not belong to the W-closure of $T$, then the sequent $\Gamma_{n+1} \supset \Delta_{n+1}$ is either $F_{m}, \Gamma_{n} \supset \Delta_{n}$ or $\Gamma_{n} \supset F_{m}, \Delta_{n}$.

(D.4) If $n=3 m+1$ and $\Gamma_{n} \supset \Delta_{n}$ does not belong to the W-closure of $T$, then the sequent $\Gamma_{n+1} \supset \Delta_{n+1}$ is determined by the rightmost formula of $\Delta_{n}$.

(D.4.1) If $\Delta_{n}$ is $\Delta_{n}^{\prime}, A$ for some atomic $A$, then $\Gamma_{n+1} \supset \Delta_{n+1}$ is $\Gamma_{n} \supset A, \Delta_{n}^{\prime}$.

(D.4.2) If $\Delta_{n}$ is $\Delta_{n}^{\prime}, \neg A$, then $\Gamma_{n+1} \supset \Delta_{n+1}$ is $A, \Gamma_{n} \supset \neg A, \Delta_{n}^{\prime}$.

(D.4.3) If $\Delta_{n}$ is $\Delta_{n}^{\prime}, A \vee B$, then $\Gamma_{n+1} \supset \Delta_{n+1}$ is $\Gamma_{n} \supset A \vee B, \Delta_{n}^{\prime}, A, B$.

(D.4.4) If $\Delta_{n}$ is $\Delta_{n}^{\prime}, A \wedge B$, then $\Gamma_{n+1} \supset \Delta_{n+1}$ is either $\Gamma_{n} \supset A \wedge B, \Delta_{n}^{\prime}, A$ or $\Gamma_{n} \supset A \wedge B, \Delta_{n}^{\prime}, B$.

(D.4.5) If $\Delta_{n}$ is $\Delta_{n}^{\prime}, \exists x A(x)$, then $\Gamma_{n+1} \supset \Delta_{n+1}$ is $\Gamma_{n} \supset \exists x A(x), \Delta_{n}^{\prime}, A\left(t_{i}\right)$, where $i$ is the least number so that $A\left(t_{i}\right)$ is not an element of $\operatorname{set}\left(\Delta_{n}^{\prime}\right)$.

(D.4.6) If $\Delta_{n}$ is $\Delta_{n}^{\prime}, \forall x A(x)$, then $\Gamma_{n+1} \supset \Delta_{n+1}$ is $\Gamma_{n} \supset \forall x A(x), \Delta_{n}^{\prime}, A\left(s_{i}\right)$, where $i$ is the least number so that $s_{i}$ does not occur in $\Gamma_{n} \supset \Delta_{n}$.

(D.5) If $n=3 m+2$ and $\Gamma_{n} \supset \Delta_{n}$ does not belong to the W-closure of $T$, then the sequent $\Gamma_{n+1} \supset \Delta_{n+1}$ is determined by the rightmost formula of $\Gamma_{n}$ analogous to D.4.1-D.4.6; the details are left to the reader.

D-chains are formed inversely to the logical rules and cut rules. The definition clauses (D.4) and (D.5) deal with the logical rules for introduction of connectives and quantifiers on the right- and left-hand side of a sequent, respectively. The clauses (D.3) take care of the cuts so that all possible cut formulas are treated.

Our next step is to prove a principal syntactic lemma and a principal semantic lemma. Then the strong completeness result is an immediate consequence of these two lemmas.

Lemma 8 (Principal syntactic lemma). Let $T$ be an $\mathscr{L}$ theory, and let $\Gamma \supset \Delta$ be an $\mathscr{L}$ sequent. If all $\mathrm{D}$-chains for $\Gamma \supset \Delta$ with respect to $T$ are finite, then we have $T \Vdash \Gamma \supset \Delta$.

Proof. It follows from König's lemma that there are only finitely many Dchains for $\Gamma \supset \Delta$ with respect to $T$. We let $m$ be the maximal length of these D-chains and prove by induction on $m-n$ : If $\Gamma_{n} \supset \Delta_{n}$ occurs in the $n$th place of one of these D-chains, then we have $T \Vdash \Gamma_{n} \supset \Delta_{n}$.

If $\Gamma_{n} \supset \Delta_{n}$ is the last sequent of a D-chain for $\Gamma \supset \Delta$ with respect to $T$, then the assertion follows by some applications of the structural rules. Otherwise, $\Gamma_{n} \supset \Delta_{n}$ does not belong to the W-closure of $T$. Therefore, if $n=3 m$ for some $m$, the sequent $F_{m}, \Gamma_{n} \supset \Delta_{n}$ and the sequent $\Gamma_{n} \supset F_{m}, \Delta_{n}$ follow upon $\Gamma_{n} \supset \Delta_{n}$. Hence, the induction hypothesis implies that

$$
T \Vdash F_{m}, \Gamma_{n} \supset \Delta_{n} \quad \text { and } \quad T \Vdash \Gamma_{n} \supset F_{m}, \Delta_{n}
$$

By means of some structural rules and a cut with cut formula $F_{m}$ one, therefore, immediately obtains $T \Vdash \Gamma_{n} \supset \Delta_{n}$. If $n$ is of the form $3 m+1$ or $3 m+2$ for some $m$, then we proceed in a similar way.

We have proved, in particular, that $T \Vdash \Gamma_{0} \supset \Delta_{0}$. By definition clause (D.1) of the definition of $\mathrm{D}$-chains this implies the principal syntactic lemma. 
Lemma 9 (Principal semantic lemma). Let $T$ be an $\mathscr{L}$ theory, and let $\Gamma \supset \Delta$ be an $\mathscr{L}$ sequent. If there exists an infinite $\mathrm{D}$-chain for $\Gamma \supset \Delta$ with respect to $T$, then there exists a strong Schüte valuation $V$ so that $V \vDash_{s} T$ and $V \nvdash_{s} \Gamma \supset \Delta$.

Proof. Let $\Gamma_{0} \supset \Delta_{0}, \Gamma_{1} \supset \Delta_{1}, \ldots$ be an infinite D-chain for $\Gamma \supset \Delta$ with respect to $T$ and define

$$
M_{l}:=\bigcup_{i \in \omega} \operatorname{set}\left(\Gamma_{i}\right) \text { and } M_{r}:=\bigcup_{i \in \omega} \operatorname{set}\left(\Delta_{i}\right) \text {. }
$$

In a first step we collect some important closure properties of the sets $M_{l}$ and $M_{r}$ which are carried over more or less directly from the characteristics of D-chains:

(P.1) If the formula $\neg A$ belongs to $M_{r}$, then $A$ belongs to $M_{l}$; if the formula $\neg A$ belongs to $M_{l}$, then $A$ belongs to $M_{r}$.

(P.2) If the formula $A \vee B$ belongs to $M_{r}$, then $A$ and $B$ belong to $M_{r}$; if the formula $A \vee B$ belongs to $M_{l}$, then $A$ or $B$ belong to $M_{l}$.

(P.3) If the formula $A \wedge B$ belongs to $M_{r}$, then $A$ or $B$ belong to $M_{r}$; if the formula $A \wedge B$ belongs to $M_{l}$, then $A$ and $B$ belong to $M_{l}$.

(P.4) If the formula $\exists x A(x)$ belongs to $M_{r}$, then all $A\left(t_{i}\right)$ belong to $M_{r}$; if the formula $\exists x A(x)$ belongs to $M_{l}$, then there is a free variable $u$ so that $A(u)$ belongs to $M_{l}$.

(P.5) If the formula $\forall x A(x)$ belongs to $M_{r}$, then there is a free variable $u$ so that $A(u)$ belongs to $M_{r}$; if the formula $\forall x A(x)$ belongs to $M_{l}$, then all $A\left(t_{i}\right)$ belong to $M_{l}$.

(P.6) For all $\mathscr{L}$ formulas $A$ we have $A \in M_{l}$ or $A \in M_{r}$.

Observe, however, that it may well happen that a formula $A$ belongs to $M_{l}$ as well as to $M_{r}$. Now we introduce the three-valued valuation $V$ for $\mathscr{L}$ by defining for all $\mathscr{L}$ formulas $A$ :

$$
V(A):= \begin{cases}\mathbf{t}, & \text { if } A \notin M_{r}, \\ \mathbf{f}, & \text { if } A \notin M_{l}, \\ \mathbf{u}, & \text { if } A \in M_{r} \text { and } A \in M_{l} .\end{cases}
$$

It follows from properties P.1-P.5 that $V$ is a strong Schütte valuation. Even more, since $\operatorname{set}(\Gamma) \subset M_{l}$ and $\operatorname{set}(\Delta) \subset M_{r}$, one also obtains that $V(A) \neq \mathbf{f}$ for all $A$ in $\Gamma$ and $V(B) \neq \mathbf{t}$ for all $B$ in $\Delta$. This means that $V$ is not a strong model of $\Gamma \supset \Delta$.

It remains to show that $V$ is a strong model of $T$. To do this, take an arbitrary element $\Pi \supset \Sigma$ of $T$. It follows that we have $\operatorname{set}(\Pi) \not \subset \operatorname{set}\left(\Gamma_{m}\right)$ or $\operatorname{set}(\Sigma) \not \subset \operatorname{set}\left(\Delta_{m}\right)$ for all $m$, since the given D-chain is infinite. Hence, there exists an $A$ in $\Pi$ which does not belong to $M_{l}$ or a $B$ in $\Sigma$ which does not belong to $M_{r}$. In the first case we obtain $V(A)=\mathbf{f}$, and the second case implies $V(B)=\mathbf{t}$. In both cases we, therefore, have $V \vDash_{s} \Pi \supset \Sigma$. This proves that $V$ is a strong model of $T$.

THEOREM 10 (Strong completeness). We have for all $\mathscr{L}$ theories $T$ and all $\mathscr{L}$ sequents $\Gamma \supset \Delta$ :

$$
T \vDash_{s} \Gamma \supset \Delta \Rightarrow T \Vdash \Gamma \supset \Delta .
$$

Proof. Assume that $T \vDash_{s} \Gamma \supset \Delta$. Then the principal semantic lemma implies that all D-chains for $\Gamma \supset \Delta$ with respect to $T$ are finite. Hence, the principal syntactic lemma permits us to conclude that $T \Vdash \Gamma \supset \Delta$. 
COROLlary 11. We have for all $\mathscr{L}$ theories $T$ and all $\mathscr{L}$ sequents $\Gamma \supset \Delta$ :

$$
T \Vdash \Gamma \supset \Delta \Leftrightarrow T \vDash_{s} \Gamma \supset \Delta .
$$

$\S 4$. Syntactical considerations. The main emphasis of this section is put on some elementary syntactical properties of the identity-free sequent calculus and the striking symmetries between cut-free and identity-free provability. The basic idea is to disconnect the positive and negative occurrences of relation symbols and to provide asymmetrical interpretations of cut-free and identity-free proofs. This approach to cut-free systems is well known and presented for example in the textbook by Girard, Lafont, and Taylor [2].

Let $\mathscr{L}^{*}$ be the first-order language which is associated to $\mathscr{L}$ by replacing each $n$-ary relation symbol $R$ of $\mathscr{L}$ by two different $n$-ary relation symbols $R^{\mathscr{P}}$ and $R^{\mathscr{N}}$; the variables, function symbols and all the other ingredients of the language are not affected. The translations $A^{\mathscr{P}}$ and $A^{\mathscr{N}}$ of $\mathscr{L}$ formulas are then inductively defined as follows:

(1) If $A$ is the atomic formula $R\left(a_{1}, \ldots, a_{n}\right)$, then $A^{\mathscr{P}}$ is $R^{\mathscr{P}}\left(a_{1}, \ldots, a_{n}\right)$ and $A^{\mathscr{N}}$ is $R^{\mathscr{N}}\left(a_{1}, \ldots, a_{n}\right)$.

(2) If $A$ is the formula $\neg B$, then $A^{\mathscr{P}}$ is $\neg B^{\mathscr{N}}$ and $A^{\mathscr{N}}$ is $\neg B^{\mathscr{P}}$.

(3) If $A$ is the formula $(B \vee C)$, then $A^{\mathscr{P}}$ is $\left(B^{\mathscr{P}} \vee C^{\mathscr{P}}\right)$ and $A^{\mathscr{N}}$ is $\left(B^{\mathscr{N}} \vee C^{\mathscr{N}}\right)$.

(4) If $A$ is the formula $(B \wedge C)$, then $A^{\mathscr{P}}$ is $\left(B^{\mathscr{P}} \wedge C^{\mathscr{P}}\right)$ and $A^{\mathscr{N}}$ is $\left(B^{\mathscr{N}} \wedge C^{\mathscr{N}}\right)$.

(5) If $A$ is the formula $\exists x B(x)$, then $A^{\mathscr{P}}$ is $\exists x B^{\mathscr{P}}(x)$ and $A^{\mathscr{N}}$ is $\exists x B^{\mathscr{N}}(x)$.

(6) If $A$ is the formula $\forall x B(x)$, then $A^{\mathscr{D}}$ is $A x B^{\mathscr{P}}(x)$ and $A^{\mathscr{N}}$ is $\forall x B^{\mathscr{N}}(x)$.

This means that the $\mathscr{L}^{*}$ formula $A^{\mathscr{P}}$ results from the $\mathscr{L}$ formula $A$ by replacing all positive occurrences of the relation symbols $R$ by $R^{\mathscr{P}}$ and the negative ones by $R^{\mathscr{N}}$. Accordingly, the $\mathscr{L}^{*}$ formula $A^{\mathscr{N}}$ is obtained from the $\mathscr{L}$ formula $A$ by replacing all positive occurrences of the relation symbols $R$ by $R^{\mathscr{N}}$ and the negative ones by $R^{\mathscr{P}}$.

If $\Gamma$ is the finite sequence $A_{1}, \ldots, A_{n}$ of $\mathscr{L}$ formulas, then $\Gamma^{\mathscr{D}}$ stands for the sequence $A_{1}^{\mathscr{P}}, \ldots, A_{n}^{\mathscr{P}}$ and $\Gamma^{\mathscr{N}}$ for the sequence $A_{1}^{\mathscr{N}}, \ldots, A_{n}^{\mathscr{N}}$. We obtain the *translation of an $\mathscr{L}$ sequent $\Gamma \supset \Delta$ by writing $(\Gamma \supset \Delta)^{*}$ for the $\mathscr{L}^{*}$ sequent $\Gamma^{\mathscr{N}} \supset \Delta^{\mathscr{P}}$. Finally, if $T$ is an $\mathscr{L}$ theory, then $T^{*}$ denotes the $\mathscr{L}^{*}$ theory which consists of the $*$-translations of the elements of $T$.

So far there are no axioms or rules which connect the relation symbols $R^{\mathscr{P}}$ and $R^{\mathscr{N}}$, and they may be treated as completely independent from each other. This will be changed by the following weak identity axioms and weak cut rules which tie the formulas $A^{\mathscr{P}}$ and $A^{\mathcal{N}}$ together.

WEAK IDENTITY AXIOMS. For all finite sequences $\Gamma$ and $\Delta$ of $\mathscr{L}^{*}$ formulas and all atomic $\mathscr{L}$ formulas $A$ :

$$
\Gamma, A^{\mathscr{N}} \supset \Delta, A^{\mathscr{D}} \quad(\mathrm{w}-\mathrm{i}) \text {. }
$$

WEAK CUT RULES. For all finite sequences $\Gamma$ and $\Delta$ of $\mathscr{L}^{*}$ formulas and all $\mathscr{L}$ formulas $A$ :

$$
\frac{\Gamma \supset \Delta, A^{\mathscr{P}} \Gamma, A^{\mathscr{N}} \supset \Delta}{\Gamma \supset \Delta} \quad(\mathrm{w}-\mathrm{c}) .
$$

The weak identity axioms state that atomic formulas $A^{\mathscr{N}}$ imply the formulas $A^{\mathscr{P}}$, i.e. that a positive occurrence of a relation symbol is at least as strong a negative 
occurrence of the same symbol. The weak cut rules express the converse situation: $A^{\mathscr{P}}$ implies $A^{\mathscr{N}}$ and so the negative version of an $\mathscr{L}$ formula is at least as strong as the positive version.

In the following we study the effect of having either the weak identity axioms or the weak cut rules at our disposal. The first obvious observation says that the weak identity axioms can be lifted from atomic formulas to arbitrary formulas by cut- and identity-free proofs. This simple fact is shown by induction on the rank of $A$.

Lemma 12. We have for all $\mathscr{L}$ formulas $A$ and $\mathscr{L}^{*}$ theories $T$ :

$$
T+(\mathrm{w}-\mathrm{i}) \vdash_{0} A^{\mathscr{N}} \supset A^{\mathscr{P}} .
$$

Now we turn to some properties of the weak identity axioms and their specific role in connection with cut-free and identity-free derivations. The first result, Lemma 13, says that the identity axioms are not needed provided that one works in cut-free environment, and the second result, Corollary 15, states that cuts are superfluous in an identity-free surrounding.

LEMma 13. We have for all $\mathscr{L}$ theories $T$ and all $\mathscr{L}$ sequents $\Gamma \supset \Delta$ :

$$
T^{*}+(\mathrm{w}-\mathrm{i}) \vdash_{0}(\Gamma \supset \Delta)^{*} \Leftrightarrow T^{*}+(\mathrm{w}-\mathrm{i}) \vdash_{0}(\Gamma \supset \Delta)^{*}
$$

The direction from right to left is obvious; the direction from left to right is proved by induction on the length of the derivation. In the second case one has only to observe that all sequents which occur in a cut-free derivation of $(\Gamma \supset \Delta)^{*}$ from $T^{*}+(\mathrm{w}-\mathrm{i})$ are of the form $\Pi^{\mathscr{N}} \supset \Sigma^{\mathscr{P}}$. Hence they are no identity axioms.

Lemma 14. Let $T$ be an $\mathscr{L}$ theory, and let $\Gamma \supset \Delta$ be an $\mathscr{L}^{*}$ sequent so that

$$
T^{*}+(\mathrm{w}-\mathrm{i}) \Vdash \Gamma \supset \Delta .
$$

Then there exists an $\mathscr{L}$ sequent $\Pi \supset \Sigma$ so that $\operatorname{set}\left(\Pi^{\mathscr{N}}\right) \subset \operatorname{set}(\Gamma), \operatorname{set}\left(\Sigma^{\mathscr{P}}\right) \subset \operatorname{set}(\Delta)$ and

$$
T^{*}+(\mathrm{w}-\mathrm{i}) \Vdash_{0}(\Pi \supset \Sigma)^{*}
$$

Proof. Again we proceed by induction on the length of the derivation of $\Gamma \supset \Delta$. For elements of $T^{*}$ and the weak identity axioms the assertion is trivially satisfied. Otherwise, $\Gamma \supset \Delta$ is the conclusion of a structural rule, a logical rule, or a cut. In the first two cases we apply the induction hypothesis to the premise(s) of this rule and apply it again, if necessary. In the third case there is an $\mathscr{L}^{*}$ formula $A$ so that

$$
\begin{aligned}
& T^{*}+(\mathrm{w}-\mathrm{i}) \Vdash \Gamma \supset \Delta, A, \\
& T^{*}+(\mathrm{w}-\mathrm{i}) \Vdash \Gamma, A \supset \Delta,
\end{aligned}
$$

and the corresponding derivations are shorter than the derivation of $\Gamma \supset \Delta$. We 
apply the induction hypothesis to (1) and (2) and obtain $\mathscr{L}$ sequents $\Pi_{1} \supset \Sigma_{1}$ and $\Pi_{2} \supset \Sigma_{2}$ with the following properties:

$$
\begin{gathered}
\operatorname{set}\left(\Pi_{1}^{\mathscr{N}}\right) \subset \operatorname{set}(\Gamma) \quad \text { and } \quad \operatorname{set}\left(\Sigma_{1}^{\mathscr{D}}\right) \subset \operatorname{set}(\Delta, A), \\
T^{*}+(\mathrm{w}-\mathrm{i}) \Vdash_{0}\left(\Pi_{1} \supset \Sigma_{1}\right)^{*}, \\
\operatorname{set}\left(\Pi_{2}^{\mathscr{N}}\right) \subset \operatorname{set}(\Gamma, A) \quad \text { and } \quad \operatorname{set}\left(\Sigma_{2}^{\mathscr{P}}\right) \subset \operatorname{set}(\Delta), \\
T^{*}+(\mathrm{w}-\mathrm{i}) \Vdash_{0}\left(\Pi_{2} \supset \Sigma_{2}\right)^{*} .
\end{gathered}
$$

Since the formula $A$ cannot belong to $\Sigma_{1}^{\mathscr{P}}$ and $\Pi_{2}^{\mathscr{N}}$ this implies the assertion with $\Pi_{1} \supset \Sigma_{1}$ or $\Pi_{2} \supset \Sigma_{2}$ as the required $\mathscr{L}$ sequent.

COROLlaRY 15. We have for all $\mathscr{L}$ theories $T$ and all $\mathscr{L}$ sequents $\Gamma \supset \Delta$ :

$$
T^{*}+(\mathrm{w}-\mathrm{i}) \Vdash(\Gamma \supset \Delta)^{*} \Leftrightarrow T^{*}+(\mathrm{w}-\mathrm{i}) \Vdash_{0}(\Gamma \supset \Delta)^{*} .
$$

Lemma 13 and Corollary 15 must be seen in combination with the following theorem. It provides a nice description of the connections between cut-free proofs of $\mathscr{L}$ sequents and the proofs of their $*$-translations by means of the weak identity axioms.

THEOREM 16. We have for all $\mathscr{L}$ theories $T$ and all $\mathscr{L}$ sequents $\Gamma \supset \Delta$ :

$$
T \vdash_{0} \Gamma \supset \Delta \Leftrightarrow T^{*}+(\mathrm{w}-\mathrm{i}) \vdash_{0}(\Gamma \supset \Delta)^{*} .
$$

Both directions of this theorem are proved by straightforward indiction on the length of the derivations. The weak identity axioms are needed to take care of the identity axioms under the $*$-translations. Just to sum up, we have the following theorem.

THEOREM 17. Let $T$ be an $\mathscr{L}$ theory, and let $\Gamma \supset \Delta$ be an $\mathscr{L}$ sequent. Then $T \vdash_{0} \Gamma \supset \Delta$ is equivalent to each of the following three assertions:

$$
T^{*}+(\mathrm{w}-\mathrm{i}) \vdash_{0}(\Gamma \supset \Delta)^{*}, \quad T^{*}+(\mathrm{w}-\mathrm{i}) \Vdash(\Gamma \supset \Delta)^{*}, \quad T^{*}+(\mathrm{w}-\mathrm{i}) \vdash_{0}(\Gamma \supset \Delta)^{*} .
$$

The following remarks concern the weak cut rules and their relationship to identity free derivations. They are symmetric versions of the results about the weak identity axioms above.

Lemma 18. We have for all $\mathscr{L}$ theories $T$ and all $\mathscr{L}$ sequents $\Gamma \supset \Delta$ :

$$
T^{*}+(\mathrm{w}-\mathrm{c}) \vdash_{0}(\Gamma \supset \Delta)^{*} \Leftrightarrow T^{*}+(\mathrm{w}-\mathrm{c}) \vdash_{0}(\Gamma \supset \Delta)^{*} .
$$

As in the case of Lemma 13, the direction from right to left is obvious, and the direction from left to right is proved by induction on the length of the derivation. Again there are no problems since all sequents which occur in a cut-free derivation of $(\Gamma \supset \Delta)^{*}$ from $T^{*}+(\mathrm{w}-\mathrm{c})$ are sequents $\Pi^{\mathscr{N}} \supset \Sigma^{\mathscr{P}}$. Hence they are no identity axioms. 
Lemma 19. Let $T$ be an $\mathscr{L}$ theory, and let $\Gamma \supset \Delta$ be an $\mathscr{L}^{*}$ sequent so that

$$
T^{*}+(\mathrm{w}-\mathrm{c}) \Vdash \Gamma \supset \Delta .
$$

Then there exists an $\mathscr{L}$ sequent $\Pi \supset \Sigma$ so that $\operatorname{set}\left(\Pi^{\mathscr{N}}\right) \subset \operatorname{set}(\Gamma), \operatorname{set}\left(\Sigma^{\mathscr{D}}\right) \subset \operatorname{set}(\Delta)$, and

$$
T^{*}+(\mathrm{w}-\mathrm{c}) \vdash_{0}(\Pi \supset \Sigma)^{*}
$$

The proof of this lemma is essentially the same as the proof of Lemma 14 so that it can be omitted. We immediately turn to the following straightforward corollary.

COROLlaRy 20. We have for all $\mathscr{L}$ theories $T$ and all $\mathscr{L}$ sequents $\Gamma \supset \Delta$ :

$$
T^{*}+(\mathrm{w}-\mathrm{c}) \Vdash(\Gamma \supset \Delta)^{*} \Leftrightarrow T^{*}+(\mathrm{w}-\mathrm{c}) \Vdash_{0}(\Gamma \supset \Delta)^{*} .
$$

In a final step we complete the picture and turn to a theorem which exhibits the relationship between identity-free probability and provability of the $*$-translations using weak cuts. It is obvious that one obtains both directions by induction on the length of the derivations and that the cut rules reduce to weak cut rules under the $*$-translations.

THEOREM 21. We have for all $\mathscr{L}$ theories $T$ and all $\mathscr{L}$ sequents $\Gamma \supset \Delta$ :

$$
T \Vdash \Gamma \supset \Delta \Leftrightarrow T^{*}+(\mathrm{w}-\mathrm{c}) \Vdash_{0}(\Gamma \supset \Delta)^{*} .
$$

It remains to sum up the above observations about identity-free provability and weak cut rules. By doing this we obtain the following result which is the symmetric version of Theorem 17.

THEOREM 22. Let $T$ be an $\mathscr{L}$ theory, and let $\Gamma \supset \Delta$ be an $\mathscr{L}$ sequent. Then $T \Vdash \Gamma \supset \Delta$ is equivalent to each of the following three assertions:

$$
T^{*}+(\mathrm{w}-\mathrm{c}) \vdash_{0}(\Gamma \supset \Delta)^{*}, \quad T^{*}+(\mathrm{w}-\mathrm{c}) \Vdash(\Gamma \supset \Delta)^{*}, \quad T^{*}+(\mathrm{w}-\mathrm{c}) \vdash_{0}(\Gamma \supset \Delta)^{*} .
$$

Final remark. This finishes our considerations concerning the interplay between identity axioms and cut rules. Theorem 4 and Corollary 11 show the strong symmetries between cut-free and identity-free provability on the semantic level. The syntactic symmetries between cut-free and identity-free derivations are made clear by Theorem 17 and Theorem 22 and the proofs of these results. In deductive approaches to logic programming one is often interested in sequent calculi extended by so called program rules and in their cut-free and identity-free derivations. It is possible to exhibit analogous symmetries for such systems (cf., e.g., Jäger [4]).

\section{REFERENCES}

[1] J.-Y. Girard, Proof theory and logical complexity, Volume I, Bibliopolis, Napoli, 1987.

[2] J.-Y. GIRARd, YVes LAFONT AND P. TAYlor, Proofs and types, Cambridge Tracts in Theoretical Computer Science, vol. 7, Cambridge University Press, Cambridge, 1989.

[3] G. JÄGER, Some proof-theoretic aspects of logic programming, Logic and algebra of specification (F. L. Bauer, W. Brauer, and H. Schwichtenberg, editors), Springer, Berlin, 1993.

[4] - A deductive approach to logic programming, Research Report Universität Bern (1993). 
[5] K. Kunen, Negation in logic programming, Journal of Logic Programming, vol. 4 (1987).

[6] K. SChütTE, Proof theory, Springer, Berlin, 1977.

[7] R. F. STÄRK, A complete axiomatization of the three-qalued completion of logic programs, Journal of Logic and Computation, vol. 1 (1991).

[8] G. TAKeuti, Proof theory, (2nd edition), North-Holland, Amsterdam, 1987.

\author{
INSTITUTE FÜR THEORETISCHE INFORMATIK \\ ETH ZÜRICH \\ CH-8092 ZÜRICH, SWITZERLAND
}

E-mail: hoesli@inf.ethz.ch

\title{
INSTITUT FÜR INFORMATIK UND ANGEWANDTE MATHEMATIK UNIVERSITÄT BERN \\ CH-3012 BERN, SWIZTERLAND
}

E-mail: jaeger@iam.unibe.ch 